\title{
Analysis of polymorphisms in the 3 ' untranslated region of the LDL receptor gene and their effect on plasma cholesterol levels and drug response
}

\author{
WEI CHEN $^{1 *}$, SHUKUI WANG ${ }^{2 *}$, YULING MA $^{2}$, YUE ZHOU $^{1}$, HAIYAN LIU $^{1}$, PAVEL STRNAD $^{1}$, \\ FREDRIC B. KRAEMER ${ }^{1}$, RONALD M. KRAUSS ${ }^{3}$ and JINGWEN LIU ${ }^{1}$ \\ ${ }^{1}$ VA Palo Alto Health Care System, Palo Alto, CA, USA; ${ }^{2}$ Nanjing First Hospital, Nanjing Medical University, \\ Nanjing, P.R. China; ${ }^{3}$ Children's Hospital Oakland Research Institute, Oakland, CA, USA
}

Received October 30, 2007; Accepted December 23, 2007

\begin{abstract}
The proximal section of the $3^{\prime}$ untranslated region (3'UTR) of LDL receptor (LDLR) mRNA contains important regulatory sequences that control the messenger stability and mediate the cholesterol-lowering drug berberine (BBR)induced increase in LDLR mRNA half-life. In the present study, we examined whether single nucleotide polymorphisms (SNPs) within this region cause a predisposition to the development of coronary heart disease (CHD) and whether they affect the response to BBR treatment. Genomic DNAs were isolated from peripheral blood of a Chinese cohort of 103 normolipidemic subjects and 94 hyperlipidemic CHD patients. The 1.1-kb proximal fragment of LDLR mRNA 3'UTR was PCR-amplified and sequenced. Six SNPs were detected within this region. Among them, the presence of SNP1 and SNP6 in both study groups showed complete association $\left(r^{2}=1\right)$. The frequency of individual SNPs and genotypes did not differ between CHD patients and normolipidemic individuals. Allelic variations did not correlate with total and LDL-cholesterol levels. To examine the effects of genetic variations in $3^{\prime} \mathrm{UTR}$ on BBR treatment, entire 2.5-kb regions of 3'UTR from three common SNP haplotypes were cloned into a luciferase reporter and the reporter constructs were transfected into HepG2 cells. The expression of reporter genes carrying different haplotypes of LDLR 3'UTR was increased to a similar extent upon BBR treatment. Taken together, these findings suggest that the 3'UTR LDLR polymorphisms commonly found in the
\end{abstract}

Correspondence to: Dr Jingwen Liu (154P), VA Palo Alto Health Care System, 3801 Miranda Avenue, Palo Alto, CA 94304, USA

E-mail: jingwen.liu@med.va.gov

${ }^{*}$ Contributed equally

Key words: LDL receptor, 3 ' untranslated region, single nucleotide polymorphism, berberine, mRNA stability, genetic variants
Chinese population do not cause a predisposition to the development of CHD, nor do they affect the plasma lipid levels or the cholesterol-lowering effect of BBR.

\section{Introduction}

Coronary heart disease (CHD) is the major cause of morbidity and mortality in the western population. Elevated plasma LDL-cholesterol (LDL-c) levels directly contribute to the development of atherosclerosis and CHD. In humans, more than $70 \%$ of LDL-c is removed from plasma by LDL receptor (LDLR)-mediated uptake in the liver. Hence, the expression level of hepatic LDLR directly influences plasma cholesterol levels. Mutations in different coding regions of the LDLR gene reduce receptor activity and give rise to the clinical phenotype of heterozygous familial hypercholesterolemia that is characterized by increased plasma levels of total cholesterol (TC) and LDL-c, tendinous xanthomata, and premature CHD (1-3). In addition to mutations in coding regions, mutations in the $5^{\prime}$ promoter regions of the LDLR gene have been identified in hypercholesterolemic patients (4-7). These mutations compromise promoter activity and reduce LDLR expression $(6,8)$.

LDLR gene expression is regulated at the transcriptional as well as at the post-transcriptional level by changes in mRNA stability $(9,10)$, which is primarily controlled by regulatory sequences present in the 2.5 -kb-long stretch of the 3' untranslated region (3'UTR). Within the 3'UTR, three mRNA destabilizing elements known as AU-rich elements (AREs) have been identified that are responsible for the rapid turnover rate of LDLR mRNA $(10,11)$. Recently, we identified berberine (BBR), a compound isolated from a Chinese herb, as a novel inducer of hepatic LDLR expression $(12,13)$. We showed that in two human hepatoma-derived cell lines BBR strongly increases LDLR mRNA and protein expression. Mechanistic studies revealed that BBR upregulates LDLR expression by extending the half-life of LDLR mRNA without affecting gene transcription. This action is mediated through a 905-bp 5' proximal section of the LDLR 3'UTR that contains both negative regulatory elements (AREs) and the speculated positive regulatory sequences. 
In a placebo-controlled clinical study we showed that oral administration of BBR in 32 hypercholesterolemic patients of a daily dose of $1 \mathrm{~g}$ for 3 months reduced serum cholesterol by $29 \%$, triglycerides (TG) by $35 \%$, and LDL-c by $25 \%$ without affecting serum HDL-c levels (12). Although this involved a small number of patients, it provided evidence that the regulation of LDLR mRNA stability can have a profound effect on plasma cholesterol levels.

Given the functional importance of the 3'UTR of LDLR, we tested whether single nucleotide polymorphisms (SNPs) within this region are associated with altered plasma cholesterol and LDL-c levels and whether they caused a predisposition to CHD development in a cohort of normolipidemic and hypercholesterolemic Chinese subjects. In addition, we analyzed whether the genetic variants described affect response to BBR treatment.

\section{Subjects and methods}

Subjects and DNA isolation. Peripheral blood samples were obtained from a Chinese cohort of 103 healthy subjects with normal cholesterol levels and 94 hypercholesterolemic patients diagnosed with CHD at Nanjing First Hospital, Nanjing Medical University, Nanjing, P.R. China. The diagnosis of CHD was based on the internationally accepted criteria (14). The demographic information of the study groups is described in Table I. Plasma levels of TC, LDL-c, HDL-c and TG were measured by standard biochemical procedures. Peripheral blood mononuclear cells (PBMC) were isolated from heparinized blood by Ficoll-hypaque centrifugation, washed 3 times with PBS and frozen at $-80^{\circ} \mathrm{C}$. Genomic DNA was subsequently isolated from PBMC with Puregene DNA Purification Kit (Gentra Systems, Minneapolis, $\mathrm{MN}$ ) according to the manufacturer's instructions. All participants gave informed consent.

Polymerase chain reaction (PCR). PCR was performed in a volume of $25 \mu \mathrm{l}$ containing $1 \mathrm{X}$ PCR buffer, $1 \mathrm{X}$ Taq Master PCR Enhancer, $0.125 \mathrm{mM}$ of each deoxynucleotide triphosphate, 25 pmol of each primer, and 1.25 units of platinum Taq polymerase (Eppendorf). The reactions were carried out at $94^{\circ} \mathrm{C}$ for $90 \mathrm{sec}$, then 38 cycles at $94^{\circ} \mathrm{C}$ for $20 \mathrm{sec}, 67^{\circ} \mathrm{C}$ for $40 \mathrm{sec}$, and $72^{\circ} \mathrm{C}$ for $90 \mathrm{sec}$, and a final extension at $72^{\circ} \mathrm{C}$ for $8 \mathrm{~min}$.

Genotyping the LDLR 3'UTR. The 1.1-kb 5' proximal section of the 3'UTR of the LDLR gene was amplified from genomic DNA samples by using primers PS-1F (5'-ccgctgtttacc atttgttggcag-3') and PS-2R (5'-gggaagtgaatggcttggaggc-3'). PCR products were purified by PCR Purification Kit (Qiagen, MD) and sequenced using primer SNP-1 (5'-aagtgactgaatcc ggtactcaccg-3'). All sequencing results were analyzed by the DNAStar Lasergene 6 software program. Detected SNPs in the 3'UTR were compared to those in a public SNP database (SeattleSNPs, http://pga.gs.washington.edu/). The SNP reference sequence (rs) ID numbers were obtained from dbSNP (NCBI).

DHPLC analysis. The presence of heterozygous variants in some SNPs was further confirmed by denaturing high- performance liquid chromatography (DHPLC) screening using a WAVE ${ }^{\mathrm{TM}}$ DNA Fragment Analysis System (Transgenomic Inc.). In this method, PCR-amplified fragments are first bound to a nonporous poly(styrene/divinyl-benzene) DnaSep $^{\mathrm{TM}}$ column (Transgenomic Inc.) and subsequently eluted through an increasing acetonitrile concentration (15). In contrast to their homozygote counterparts, the re-annealing of heterozygous sequences leads to a formation of heteroduplexes, which are denatured earlier. These heteroduplexes can therefore be distinguished through an earlier elution from homoduplexes, when analyzed at an appropriate temperature. At such a temperature, heterozygous variants are detected as a double peak, whereas a single peak is seen in the remaining samples. The analysis was carried out using Wavemaker ${ }^{\mathrm{TM}}$ 4.1.44 software, a 2-min quick-time detection mode and a $0.5 \%$ increase in acetonitrile concentration per min (16).

Haplotype cloning and UTR reporter construction. The entire 3'UTR 2.5-kb region was PCR-amplified using primers LDLR-1 up (5'-ctagtctagacacccatctcccagaagccactc-3') and LDLR-1lo (5'-aagtgactgaatccggtactcaccg-3') from genomic DNA samples of different haplotypes. The gel-purified PCR product was initially ligated into a pCR2.1-TOPO cloning vector (Invitrogen, Carlsbad, CA) and 8-10 colonies for each DNA sample were sequenced to verify each haplotype. For reporter construction, the $2.5-\mathrm{kb}$ fragments from three haplotypes (I, IV and V) were released from pCR2.1-TOPO vector by $X b a \mathrm{I}$ digestion and were inserted adjacent to the $3^{\prime}$ firefly luciferase coding region of the plasmid pLuc (17) at the $\mathrm{XbaI}$ site to yield pLuc-UTR-I, pLuc-UTR-IV, and pLucUTR-V. The luciferase gene transcription in plasmid pLuc is driven by a CMV promoter. All constructs were sequenced and the clones with correct insert orientation were further propagated to isolate plasmid DNA.

Transient transfection and dual luciferase reporter assays. HepG2 cells were maintained at $37^{\circ} \mathrm{C}$ in an atmosphere of $5 \% \mathrm{CO}_{2}$ in Eagle's minimum essential medium (EMEM) supplemented with $10 \%$ fetal bovine serum (FBS) and antibiotics. For transfection experiments, cells were seeded in $24-w e l l$ plates at a density of $2 \times 10^{5}$ cells/well. Cells were transfected with plasmid DNA of pLuc-UTR and the control plasmid pRL-SV40 at the ratio of 9:1 using FuGene reagent. Twenty-four hours post transfection, the medium was changed to $0.5 \%$ FBS-EMEM and cells were treated with BBR at a dose of $10 \mu \mathrm{g} / \mathrm{ml}$ or the BBR carrier DMSO $(0.1 \%)$ for $8 \mathrm{~h}$ prior to cell lysis. The activity of firefly luciferase and renilla luciferase in cell lysate was measured using the Dual Luciferase Reporter Assay System (Promega). Triplicate wells were assayed for each construct and transfection experiments were repeated 4 times.

Statistical analysis. For the characterization of study groups, the two-tailed Student's t-test was used to compare the mean values of TC, LDL-c, TG, or HDL-c between CHD and healthy individuals of the same gender without adjusting for age or use of medicine. Allele frequencies were estimated by the gene counting method. The distributions of genotypes and allele frequencies between CHD and normal control subjects were analyzed using the two-tailed Fisher's exact test. One- 

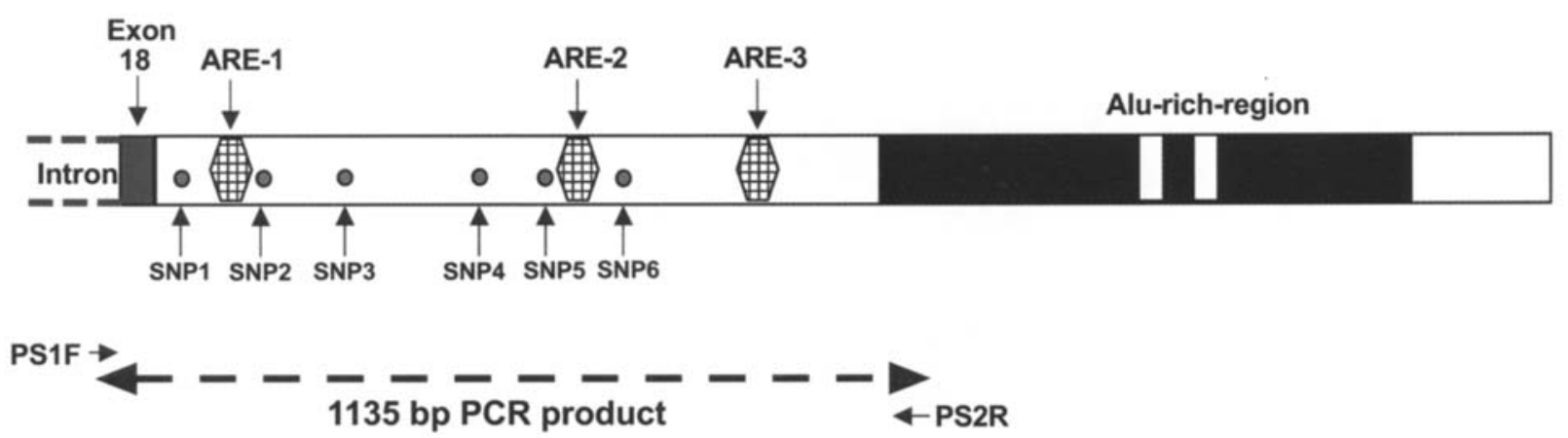

Figure 1. Schematic presentation of the human LDLR mRNA 3'UTR and the analyzed 1.1-kb PCR product. The distribution of observed SNPs and the location of the AU-rich regulatory elements (AREs) are indicated. The region was amplified using primers PS1F and PS2R.

Table I. Characterization of the study groups.

\begin{tabular}{|c|c|c|c|c|c|c|}
\hline & \multicolumn{3}{|c|}{ Male } & \multicolumn{3}{|c|}{ Female } \\
\hline & CHD $(n=44)$ & $\mathrm{HI}(\mathrm{n}=47)$ & P-value & CHD $(n=49)$ & $\mathrm{HI}(\mathrm{n}=56)$ & P-value \\
\hline Age (years) & $62.8 \pm 15.3$ & $50.5 \pm 19.1$ & 0.0011 & $62.3 \pm 10.6$ & $46.5 \pm 18.5$ & 0.0001 \\
\hline $\mathrm{TC}(\mathrm{mmol} / \mathrm{l})$ & $5.6 \pm 1.0$ & $3.5 \pm 0.6$ & 0.0001 & $5.8 \pm 0.7$ & $3.8 \pm 0.6$ & 0.0001 \\
\hline LDL-c (mmol/l) & $3.6 \pm 1.0$ & $2.1 \pm 0.5$ & 0.0001 & $3.7 \pm 0.7$ & $2.3 \pm 0.4$ & 0.0001 \\
\hline HDL-c (mmol/l) & $1.1 \pm 0.3$ & $1.1 \pm 0.3$ & 0.9999 & $1.3 \pm 0.3$ & $1.1 \pm 0.3$ & 0.0009 \\
\hline $\mathrm{TG}(\mathrm{mmol} / \mathrm{l})$ & $2.0 \pm 1.5$ & $1.0 \pm 0.8$ & 0.0001 & $2.1 \pm 1.6$ & $1.2 \pm 1.0$ & 0.0007 \\
\hline
\end{tabular}

Data are expressed as mean \pm SD. For each lipid parameter, the unpaired two-tailed Student's t-test was used to compare the mean values between coronary heart disease (CHD) patients and healthy individuals (HI) of the same gender.

way ANOVA was used to compare the mean values of TC, LDL-c, TG, or HDL-c in groups of individuals with different genotypes using InStat software version 3 (GraphPad Software, San Diego, CA). Analyses were carried out with and without adjusting for age or gender. Linkage disequilibrium analysis was determined using the ldSelect program (http://droog.gs.washington.edu/multiPopTagSelect.html).

\section{Results}

Characteristics of analyzed cohorts. We hypothesized that if SNPs in the LDLR 3'UTR lead to elevated plasma cholesterol levels, the frequency of such genetic variations would be higher in hypercholesterolemic patients than in healthy subjects, which in turn may predispose them to development of CHD. To test this hypothesis, we analyzed lipid profiles and isolated genomic DNA from blood samples in a cohort of 103 healthy individuals and 94 CHD patients with elevated cholesterol levels who were diagnosed at the Outpatient Clinic Unit of Nanjing First Hospital. Table I lists the lipid profiles of the study groups. Plasma levels of TC, LDL-c, and TG were significantly higher in both men and women in the CHD group compared to the healthy subjects. HDL-c levels did not differ between male CHD patients and healthy male subjects, while HDL-c levels in female CHD patients were slightly higher than in the healthy female subjects. The analysis results of lipid parameters were not adjusted by the mean age difference between the patients and healthy individuals.

LDLR 3'UTR genotype analyses. Fig. 1 shows a diagram of the LDLR mRNA 3'UTR. The 5' proximal $1.1-\mathrm{kb}$ section of the LDLR mRNA 3'UTR contains 3 mRNA destabilizing motifs known as AU-rich elements (AREs) (10) as well as the speculated stabilizing regulatory sequence that mediates the effect of BBR on LDLR expression $(12,13)$. To examine nucleotide polymorphisms within this region, the $1.1-\mathrm{kb}$ region was PCR-amplified from genomic DNA samples isolated from 103 healthy individuals and from 94 CHD patients and direct DNA sequencing was performed for every PCR product. We detected the 6 SNPs, including G44243A, G44332A, C44506G, G44695A, C44857T and A44964G within this region. Fig. $2 \mathrm{~A}$ shows the representative sequencing results for each SNP.

The distribution of major genotypes in both cohorts is summarized in Table II; some minor genotypes with frequencies $<6 \%$ were excluded. Thirteen CHD and 12 normal subjects were homozygous for A-G-C-A-C-G. We termed this genotype Type I-a. Eighteen CHD individuals and 10 healthy subjects differed from Type I-a at SNPs 1, 4, and 6 , where they exhibited a heterozygous sequence (i.e. sequence $\mathrm{A} / \mathrm{G}-\mathrm{G}-\mathrm{C}-\mathrm{A} / \mathrm{G}-\mathrm{C}-\mathrm{A} / \mathrm{G})$. This genotype was designated Type I-b. Type I ( $a$ and b) was the most common genotype in this Chinese cohort with prevalences of $33 \%$ and 
A.

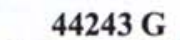

44243 G/A

SNP1

(rs 14158)
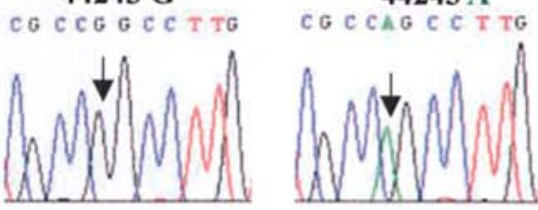

CG C CH GCC T TG

G A A EA 0 G $T=C$

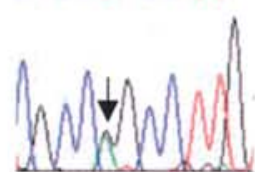

SNP2

(rs 3826810)

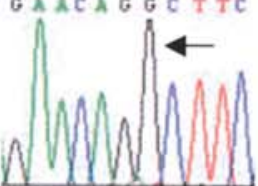

GAACAGACTTC

44332 G/A

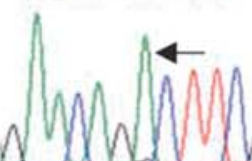

GAACA GNCTTC

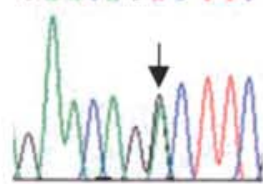

$44506 \mathrm{C}$

$44506 \mathrm{C} / \mathrm{G}$

SNP3

(rs 2738464)
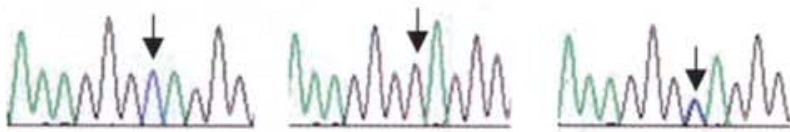

44695 G

44695 A

44695 G/A

CCCCCGAATCA

CCCCCAAATCA.

C C C C C H A A T CA

SNP4

(rs 2738465)
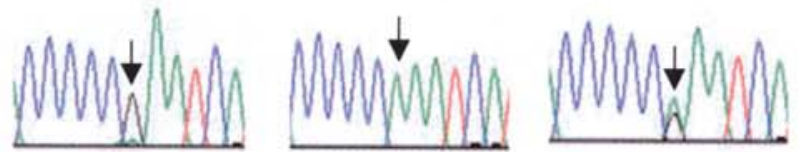

$44857 \mathrm{C}$

$44857 \mathrm{~T}$

AGTCACCGGTC

AGTCACTGGTC. IGTCACAGGTC

SNP5

(rs 1433099)
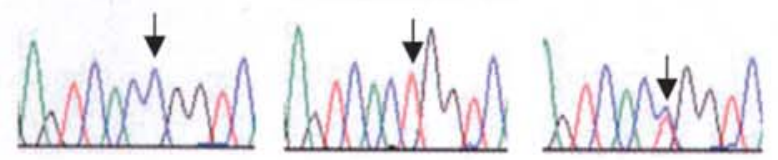

44964 A

44964 G

$44964 \mathrm{~A} / \mathrm{G}$

TGCCCATCCTC TGCCCGTCCTI TGCCCHTCCTI

SNP6

(rs 2738466)
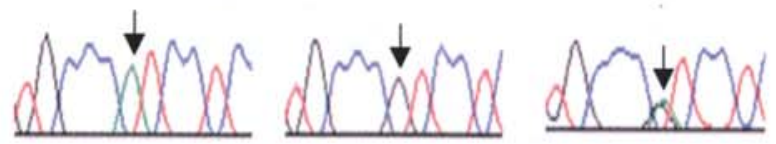

B.
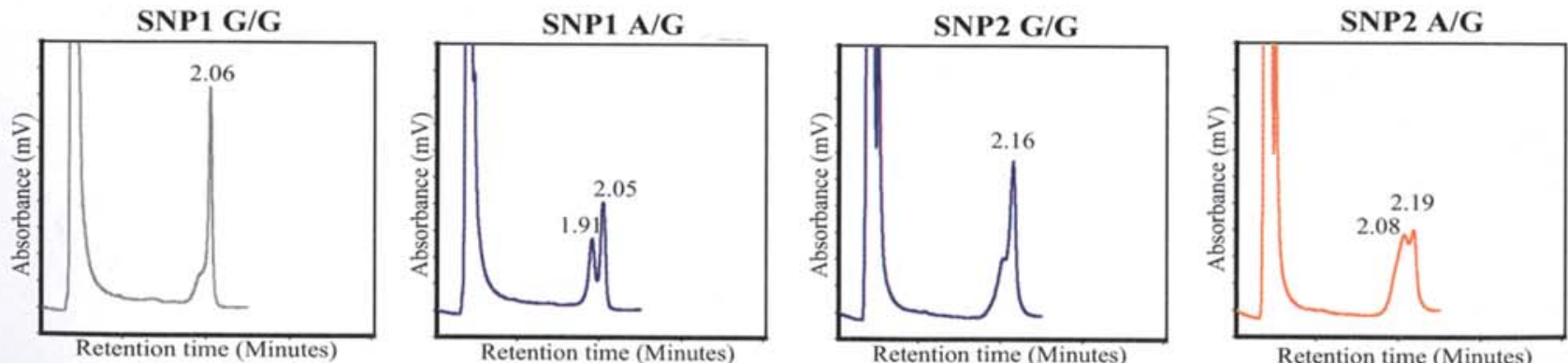

Figure 2. Identification of SNPs presented in this study. The 1.1-kb DNA fragments were PCR-amplified from different genomic DNA samples. Primer SNP-1 was used in the sequencing reaction as described in Subjects and methods. (A) The chromatogram results for a 'wild-type', 'homozygous', and 'heterozygous' allele of each SNP. (B) Examples of the denaturing high-performance liquid chromatography (DHPLC) elution patterns of amplified genomic DNA from study subjects with SNP1 and SNP2 homozygous or heterozygous alleles. 
Table II. Summary of SNP genotypes of LDLR 3'UTR in a Chinese cohort.

\begin{tabular}{|c|c|c|c|c|c|c|c|c|c|c|c|}
\hline \multirow[t]{2}{*}{ Genotype } & \multirow[t]{2}{*}{$\begin{array}{c}\text { rs14158 } \\
\text { SNP1 }\end{array}$} & \multirow[t]{2}{*}{$\begin{array}{c}\text { rs3826810 } \\
\text { SNP2 }\end{array}$} & \multirow[t]{2}{*}{$\begin{array}{l}\text { rs2738464 } \\
\text { SNP3 }\end{array}$} & \multirow[t]{2}{*}{$\begin{array}{l}\text { rs2738465 } \\
\text { SNP4 }\end{array}$} & \multirow[t]{2}{*}{$\begin{array}{l}\text { rs } 1433099 \\
\text { SNP5 }\end{array}$} & \multirow[t]{2}{*}{$\begin{array}{l}\text { rs } 2738466 \\
\text { SNP6 }\end{array}$} & \multicolumn{2}{|c|}{$\begin{array}{c}\text { CHD } \\
(n=94)\end{array}$} & \multicolumn{2}{|c|}{$\begin{array}{c}\text { HI } \\
(n=103)\end{array}$} & \multirow[t]{2}{*}{ P-value } \\
\hline & & & & & & & $\mathrm{n}$ & $\%$ & $\mathrm{n}$ & $\%$ & \\
\hline $\mathrm{I}-\mathrm{a}$ & $\mathrm{A} / \mathrm{A}$ & $\mathrm{G} / \mathrm{G}$ & $\mathrm{C} / \mathrm{C}$ & $\mathrm{A} / \mathrm{A}$ & $\mathrm{C} / \mathrm{C}$ & $\mathrm{G} / \mathrm{G}$ & 13 & 14 & 12 & 12 & 0.8 \\
\hline$I-b$ & $\mathrm{~A} / \mathrm{G}$ & $\mathrm{G} / \mathrm{G}$ & $\mathrm{C} / \mathrm{C}$ & $\mathrm{A} / \mathrm{G}$ & $\mathrm{C} / \mathrm{C}$ & $\mathrm{A} / \mathrm{G}$ & 18 & 19 & 10 & 10 & 0.1 \\
\hline II & $\mathrm{A} / \mathrm{G}$ & $\mathrm{G} / \mathrm{G}$ & $\mathrm{C} / \mathrm{G}$ & $\mathrm{A} / \mathrm{G}$ & $\mathrm{C} / \mathrm{T}$ & $\mathrm{A} / \mathrm{G}$ & 17 & 18 & 24 & 23 & 0.5 \\
\hline III & $\mathrm{A} / \mathrm{G}$ & $\mathrm{A} / \mathrm{G}$ & $\mathrm{C} / \mathrm{C}$ & $\mathrm{A} / \mathrm{A}$ & $\mathrm{C} / \mathrm{C}$ & $\mathrm{A} / \mathrm{G}$ & 10 & 11 & 11 & 11 & 1.0 \\
\hline IV-a & $\mathrm{G} / \mathrm{G}$ & $\mathrm{G} / \mathrm{G}$ & $\mathrm{G} / \mathrm{G}$ & $\mathrm{G} / \mathrm{G}$ & $\mathrm{T} / \mathrm{T}$ & $\mathrm{A} / \mathrm{A}$ & 4 & 4 & 9 & 9 & 0.3 \\
\hline IV-b & $\mathrm{G} / \mathrm{G}$ & $\mathrm{G} / \mathrm{G}$ & $\mathrm{G} / \mathrm{C}$ & $\mathrm{G} / \mathrm{G}$ & $\mathrm{T} / \mathrm{C}$ & $\mathrm{A} / \mathrm{A}$ & 9 & 10 & 7 & 7 & 0.6 \\
\hline V & $\mathrm{G} / \mathrm{G}$ & $\mathrm{G} / \mathrm{G}$ & $\mathrm{C} / \mathrm{C}$ & $\mathrm{G} / \mathrm{G}$ & $\mathrm{C} / \mathrm{C}$ & $\mathrm{A} / \mathrm{A}$ & 4 & 4 & 3 & 3 & 0.7 \\
\hline
\end{tabular}

The frequency of SNP genotypes among coronary heart disease (CHD) patients and healthy individuals (HI) was compared by using the two-tailed Fisher's exact test.

Table III. Allele frequencies of six SNPs in the study groups.

\begin{tabular}{|c|c|c|c|c|c|c|c|c|c|c|}
\hline \multirow{2}{*}{$\begin{array}{l}\text { Polymorphism } \\
\text { SNP1 }\end{array}$} & \multirow{2}{*}{$\begin{array}{c}\text { Nucleotide position } \\
44243\end{array}$} & \multirow[t]{2}{*}{ Study group } & \multicolumn{3}{|c|}{ Genotype } & \multicolumn{2}{|c|}{ Allele } & \multicolumn{2}{|c|}{ Allele frequency } & \multirow[t]{2}{*}{ P-value } \\
\hline & & & GG & GA & AA & $\mathrm{G}$ & A & $\mathrm{G}$ & A & \\
\hline & & CHD & 32 & 49 & 13 & 113 & 75 & 0.60 & 0.40 & \\
\hline & & $\mathrm{HI}$ & 36 & 55 & 12 & 127 & 79 & 0.62 & 0.38 & 0.75 \\
\hline \multirow[t]{3}{*}{ SNP2 } & 44332 & & GG & GA & $\mathrm{AA}$ & G & A & G & A & \\
\hline & & CHD & 72 & 21 & 1 & 165 & 23 & 0.88 & 0.12 & \\
\hline & & $\mathrm{HI}$ & 81 & 20 & 2 & 182 & 24 & 0.88 & 0.12 & 0.87 \\
\hline \multirow[t]{3}{*}{ SNP3 } & 44506 & & $\mathrm{CC}$ & $\mathrm{CG}$ & GG & $\mathrm{C}$ & $\mathrm{G}$ & $\mathrm{C}$ & G & \\
\hline & & CHD & 52 & 37 & 5 & 141 & 47 & 0.75 & 0.25 & \\
\hline & & HI & 46 & 46 & 11 & 138 & 68 & 0.67 & 0.33 & 0.09 \\
\hline \multirow[t]{3}{*}{ SNP4 } & 44695 & & GG & GA & AA & G & A & G & A & \\
\hline & & CHD & 19 & 51 & 24 & 89 & 99 & 0.47 & 0.53 & \\
\hline & & $\mathrm{HI}$ & 25 & 53 & 25 & 103 & 103 & 0.50 & 0.50 & 0.62 \\
\hline \multirow[t]{3}{*}{ SNP5 } & 44857 & & $\mathrm{CC}$ & $\mathrm{CT}$ & $\mathrm{TT}$ & $\mathrm{C}$ & $\mathrm{T}$ & $\mathrm{C}$ & $\mathrm{T}$ & \\
\hline & & CHD & 54 & 35 & 5 & 143 & 45 & 0.76 & 0.24 & \\
\hline & & HI & 53 & 40 & 10 & 146 & 60 & 0.71 & 0.29 & 0.25 \\
\hline \multirow[t]{3}{*}{ SNP6 } & 44964 & & $\mathrm{AA}$ & $\mathrm{AG}$ & GG & A & $\mathrm{G}$ & A & $\mathrm{G}$ & \\
\hline & & CHD & 32 & 49 & 13 & 113 & 75 & 0.60 & 0.40 & \\
\hline & & HI & 36 & 55 & 12 & 127 & 79 & 0.62 & 0.38 & 0.75 \\
\hline
\end{tabular}

CHD, coronary heart disease; HI, healthy individual. The allele frequency of each SNP between HI and CHD was compared by the twotailed Fisher's exact $2 \times 2$ test.

$22 \%$ in the two study groups, respectively. The second most abundant genotype (Type II) was homozygous at the SNP2 position but heterozygous for SNPs $1,3,4,5$, and 6 (A/G-G$\mathrm{C} / \mathrm{G}-\mathrm{A} / \mathrm{G}-\mathrm{C} / \mathrm{T}-\mathrm{A} / \mathrm{G})$. The Type III genotype (A/G-A/G-C-A$\mathrm{C}-\mathrm{A} / \mathrm{G}$ ) was homozygous for SNPs 3,4 , and 5 but heterozygous at SNPs 1, 2, and 6. This genotype represented 22\% of the Chinese cohort. We detected another homozygous genotype with the SNP pattern G-G-G-G-T-A, which was designated Type IV-a. Four CHD patients and 9 healthy controls exhibited this genotype. Nine individuals in the CHD group and 7 in the normal control groups differed from Type IV-a at SNP 3 and SNP 5 where they were heterozygous, and were termed Type IV-b (G-G-G/C-G-T/C-A). Only 4 CHD patients and 3 normal controls matched the reference sequence (NC_000019) at all six SNP sites (G-GC-G-C-A). We designated this genotype as Type V.

In addition to direct DNA sequencing, DHPLC analysis was performed to verify the heterozygous status of several 
Table IV. Analysis of linkage disequilibrium for all possible two-way comparisons among six SNPs.

\begin{tabular}{|c|c|c|c|c|c|c|c|}
\hline & & SNP1 & SNP2 & SNP3 & SNP4 & SNP5 & SNP6 \\
\hline \multirow[t]{2}{*}{ SNP1 } & CHD & $r^{2}$ & 0.09 & 0.22 & 0.60 & 0.21 & 1.00 \\
\hline & HI & & 0.08 & 0.31 & 0.62 & 0.26 & 1.00 \\
\hline \multirow[t]{2}{*}{ SNP2 } & CHD & & & 0.05 & 0.13 & 0.04 & 0.09 \\
\hline & HI & & & 0.06 & 0.13 & 0.05 & 0.08 \\
\hline \multirow[t]{2}{*}{ SNP3 } & CHD & & & & 0.37 & 0.78 & 0.22 \\
\hline & $\mathrm{HI}$ & & & & 0.49 & 0.63 & 0.31 \\
\hline \multirow[t]{2}{*}{ SNP4 } & CHD & & & & & 0.35 & 0.60 \\
\hline & HI & & & & & 0.41 & 0.62 \\
\hline \multirow[t]{2}{*}{ SNP5 } & CHD & & & & & & 0.21 \\
\hline & $\mathrm{HI}$ & & & & & & 0.26 \\
\hline
\end{tabular}

Linkage disequilibrium (LD) was determined using the ldSelect program (http://droog.gs.washington.edu/multiPopTagSelect.html). A LD value $\geq 0.8$ was considered significant.

Table V. Allele frequencies of LDLR 3'UTR in different ethnic populations.

\begin{tabular}{|c|c|c|c|c|c|c|c|}
\hline & \multirow[t]{2}{*}{ Ethnicity (n) } & SNP1 & SNP2 & SNP3 & SNP4 & SNP5 & SNP6 \\
\hline & & $\mathrm{G} / \mathrm{A}$ & G/A & $\mathrm{C} / \mathrm{G}$ & G/A & $\mathrm{C} / \mathrm{T}$ & $\mathrm{A} / \mathrm{G}$ \\
\hline Present study & CH (197) & $0.61 / 0.39$ & $0.88 / 0.12$ & $0.71 / 0.29$ & $0.49 / 0.51$ & $0.73 / 0.27$ & $0.61 / 0.39$ \\
\hline \multirow{6}{*}{ Seattle database ${ }^{b}$} & AA (62) & $0.81 / 0.19^{\mathrm{a}}$ & $0.90 / 0.10$ & $0.65 / 0.35$ & na & $0.49 / 0.51^{\mathrm{a}}$ & na \\
\hline & $\mathrm{CH} \quad(45)$ & $0.59 / 0.41$ & $0.85 / 0.15$ & $0.77 / 0.23$ & na & $0.78 / 0.22$ & na \\
\hline & ED (60) & $0.78 / 0.22^{\mathrm{a}}$ & $0.98 / 0.02^{\mathrm{a}}$ & $0.88 / 0.12^{\mathrm{a}}$ & na & $0.70 / 0.30$ & na \\
\hline & $\mathrm{HP} \quad(60)$ & $0.69 / 0.31$ & $0.96 / 0.04$ & $0.78 / 0.22$ & na & $0.81 / 0.19$ & na \\
\hline & $\mathrm{JP} \quad(45)$ & $0.54 / 0.46$ & $0.93 / 0.07$ & $0.70 / 0.30$ & na & $0.72 / 0.28$ & na \\
\hline & YB (60) & $0.83 / 0.17^{\mathrm{a}}$ & $0.88 / 0.12$ & $0.65 / 0.35$ & na & $0.38 / 0.62^{\mathrm{a}}$ & na \\
\hline \multirow[t]{4}{*}{ ARIC study ${ }^{c}$} & CC male (4967) & na & na & na & na & $0.72 / 0.28$ & $0.74 / 0.26^{\mathrm{a}}$ \\
\hline & CC female (5635) & na & na & na & na & $0.74 / 0.26$ & $0.74 / 0.26^{\mathrm{a}}$ \\
\hline & CC male (1316) & na & na & na & na & $0.48 / 0.52^{\mathrm{a}}$ & $0.82 / 0.18^{\mathrm{a}}$ \\
\hline & CC female (2181) & na & na & na & na & $0.45 / 0.55^{\mathrm{a}}$ & $0.83 / 0.17^{\mathrm{a}}$ \\
\hline
\end{tabular}

AA, African American; CH, Chinese; ED, European descent; HP, Hispanic; JP, Japanese; YB, Yoruba; CC, caucasian. ARIC, Atherosclerosis Risk in Communities. The allele frequency of each SNP described in this study was compared with previously published data from other ethnic groups using the two-tailed Fisher's exact test; ${ }^{\mathrm{P}}<0.001$. ${ }^{\mathrm{b}} \mathrm{Data}$ are cited from SeattleSNPs (http://pga.gs.washington.edu/). 'Data are derived from reference (18).

SNP sites in DNA samples (Fig. 2B). The results of DHPLC confirmed the sequencing results.

The two-tailed Fisher's exact test was used to assess the statistical difference in frequencies of each genotype between CHD patients and the normal control group. The P-values in Table II indicate that genotype frequencies were similar between CHD and normal control groups. We also compared the difference in observed allele frequencies of each single SNP between the two study groups and significant differences were not observed (Table III). These data suggest that polymorphisms in the 3'UTR region of LDLR do not cause a predisposition to CHD development.
The results of linkage disequilibrium (LD) analysis are summarized in Table IV. Among the 6 SNPs within the 3'UTR 1.1-kb region, only SNP1 and SNP6 showed complete LD $\left(r^{2}=1\right)$ in the two study groups. To the best of our knowledge, this is the first demonstration of the complete association between SNP1 and SNP6 of LDLR 3'UTR region in human population studies.

Comparisons of minor allele frequencies of this Chinese cohort with previously published data from other ethnic groups (Table V) show that the minor allele frequencies of SNP1 and SNP6 in the Chinese population are significantly higher than in African Americans (AA), and the minor allele 
Table VI. Association of plasma cholesterol levels with genotypes.

\begin{tabular}{|c|c|c|c|c|c|c|c|c|c|c|}
\hline \multirow[t]{2}{*}{ Genotype } & \multicolumn{2}{|c|}{$\mathrm{n}$} & \multicolumn{2}{|c|}{$\mathrm{TC}(\mathrm{mmol} / \mathrm{l})$} & \multicolumn{2}{|c|}{$\mathrm{TG}(\mathrm{mmol} / \mathrm{l})$} & \multicolumn{2}{|c|}{ HDL-c (mmol/l) } & \multicolumn{2}{|c|}{ LDL-c (mmol/l) } \\
\hline & CHD & $\mathrm{HI}$ & CHD & $\mathrm{HI}$ & $\mathrm{CHD}$ & HI & CHD & HI & CHD & HI \\
\hline $\mathrm{I}-\mathrm{a}$ & 13 & 12 & $5.5 \pm 0.6$ & $3.6 \pm 0.1$ & $1.8 \pm 0.9$ & $1.1 \pm 0.3$ & $1.1 \pm 0.5$ & $0.9 \pm 0.1$ & $3.5 \pm 0.7$ & $2.4 \pm 0.2$ \\
\hline $\mathrm{I}-\mathrm{b}$ & 18 & 10 & $5.5 \pm 1.1$ & $4.0 \pm 0.2$ & $1.5 \pm 1.1$ & $1.7 \pm 1.6$ & $1.3 \pm 0.2$ & $1.0 \pm 0.1$ & $3.4 \pm 0.8$ & $2.4 \pm 0.1$ \\
\hline II & 17 & 24 & $5.5 \pm 0.9$ & $3.6 \pm 0.4$ & $1.6 \pm 1.3$ & $0.9 \pm 1.0$ & $1.3 \pm 0.2$ & $1.2 \pm 0.2$ & $3.5 \pm 0.8$ & $2.2 \pm 0.2$ \\
\hline III & 10 & 11 & $5.6 \pm 1.1$ & $3.5 \pm 0.5$ & $2.2 \pm 1.8$ & $0.8 \pm 0.1$ & $1.0 \pm 0.3$ & $1.1 \pm 0.2$ & $3.7 \pm 1.1$ & $2.1 \pm 0.3$ \\
\hline IV-a & 4 & 9 & $5.1 \pm 0.8$ & $3.7 \pm 0.7$ & $1.7 \pm 1.2$ & $1.2 \pm 0.4$ & $1.3 \pm 0.4$ & $1.1 \pm 0.1$ & $3.1 \pm 0.7$ & $2.2 \pm 0.4$ \\
\hline IV-b & 9 & 7 & $5.2 \pm 0.9$ & $3.7 \pm 0.3$ & $1.6 \pm 0.8$ & $0.8 \pm 0.01$ & $1.1 \pm 0.2$ & $1.2 \pm 0.0$ & $3.3 \pm 0.9$ & $2.3 \pm 0.2$ \\
\hline
\end{tabular}

Date are expressed as mean $\pm \mathrm{SD}$. One-way ANOVA was used to compare the mean values of TC, LDL-c, TG, or HDL-c in groups of individuals with different genotypes. Analyses were carried out with and without adjusting for age and gender. Significant differences were not found.
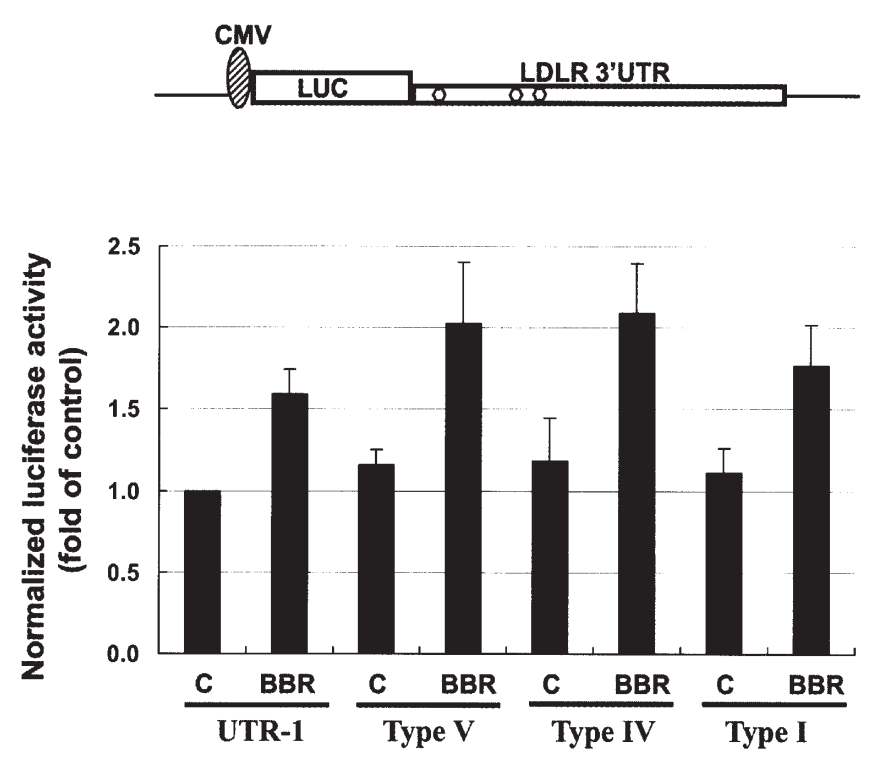

Figure 3. 3'UTR polymorphisms are not involved in the regulation of LDLR mRNA stability. The 2.5-kb LDLR 3'UTR sequences containing genotypes IV, V and I were PCR-amplified and fused to the 3 ' end of luciferase coding region (upper panel). The resulting constructs were transiently transfected into HepG2 cells along with the control plasmid pLuc-UTR-1. The plasmid pRL-SV40 expressing the renilla luciferase gene was included in each transfection for the normalization of the transfection efficiency. Transfected cells were treated with BBR for $8 \mathrm{~h}$. Cell lysates were collected at the end of treatment and firefly luciferase and renilla luciferase activities were measured. The normalized luciferase activity is expressed as the fold activity in untreated cells of pLuc-UTR-1, which is arbitrarily set as one. The data $($ mean \pm SEM) shown are derived from 4 separate transfection assays in which triplicate wells were assayed for each condition. No significant difference was observed for any of the analyzed genotypes.

frequencies of SNP1, SNP2 and SNP3 in the Chinese population are substantially higher compared to individuals of European descent.

Association of LDLR 3'UTR genotypes with lipid profiles in the study groups. It was recently reported that SNP5 and SNP6 are associated with elevated plasma LDL-c levels in Caucasians but not in African Americans (18). One-way
ANOVA analysis was conducted to compare the mean values of TC, LDL-c, TG, and HDL-c in groups of CHD patients and in healthy normal controls with different 3'UTR genotypes. The data shown in Table VI indicate that the 3'UTR genotypes were not associated with altered plasma lipid levels either in the normal group or in CHD patients in this Chinese cohort. Similar analyses were further conducted to compare the mean values of individual lipid parameters with different alleles at each SNP site. These analyses also did not detect any association of polymorphism with altered cholesterol and other lipid levels, with and without the adjustment for age or gender.

Reporter gene expression analysis of LDLR 3'UTR haplotypes in human hepatoma cells. As our previous studies using different deletion constructs localized the BBR-responsive sequence to the 1.1-kb section of LDLR 3'UTR (12), we wanted to determine whether the naturally occurring nucleotide polymorphisms within this region are associated with response to BBR treatment. To address this question, we PCR-amplified entire 3'UTR 2.5-kb DNA fragments from three common haplotypes (I, IV, and V) and inserted these fragments into a cytomegalovirus promoter-driven luciferase plasmid (pLuc). The insertion took place at the $3^{\prime}$ end of the Luc coding sequence before the SV40 polyadenylation signal (Fig. 3, upper panel). The reporters were transiently transfected into HepG2 cells along with the control plasmid pRSV-Luc that encodes the renilla luciferase gene to normalize for transfection efficiency. As a positive control for BBR treatment in these experiments, we included the previously tested plasmid pLuc-UTR-1, in which the $2.5-\mathrm{kb}$ 3'UTR was obtained from the ATCC plasmid pLDLR3 (13). Twenty-four hours after transfection, cells were treated for $8 \mathrm{~h}$ with BBR at a dose of $10 \mu \mathrm{g} / \mathrm{ml}$ or the drug carrier $(0.1 \%$ DMSO) as a control. The data presented in Fig. 3 were derived from 4 separate transfection experiments (mean \pm SEM). The basal luciferase activities of different plasmids were similar. BBR treatment resulted in an approximate 1.7-fold increase in luciferase activity in all haplotypes, indicating that these haplotypes are equally responsive to BBR treatment. 


\section{Discussion}

Genetic mutations in the LDLR gene have been studied intensively in the coding region and to some degree in the promoter region (1-7,19). A large number of these mutations are associated with elevated plasma cholesterol and LDL-c levels and give rise to clinical symptoms of severe or mild hypercholesterolemia. In addition, a number of common DNA polymorphisms have been identified in the LDLR coding region. It has been shown that some of these nucleotide variations are associated with plasma cholesterol levels in certain population groups $(20,21)$. At present, however, only a few reported studies have examined genetic polymorphisms in the LDLR 3'UTR region $(18,21)$. Our recent findings that the new cholesterol-lowering drug BBR increases LDLR expression through a mechanism involving the 3'UTR stimulated our interest in conducting this study to examine the polymorphisms within this region.

We postulated that if certain SNPs or genotypes are associated with altered plasma LDL-c levels, these SNPs/ genotypes could be found at different frequencies in healthy subjects and CHD patients, respectively. Therefore, we chose a study cohort consisting of 103 healthy subjects that had normal lipid levels and 94 CHD patients who had elevated TC and LDL-c. Our results detected 6 SNPs within this region. However, the frequency and the genotype pattern of these SNPs did not differ between normal and CHD patients, and we did not observe significant associations of genotypes with plasma cholesterol or LDL-c levels. Thus, our results suggest that these SNP sites do not influence plasma cholesterol levels or the development of CHD, at least in a Chinese population. Our results differ from those in a recent report that examined the frequency of SNP5 and SNP6 in the Atherosclerosis Risk in Communities (ARIC) study population (18). That study showed that the fully adjusted mean LDL-c level in Caucasian men with homozygous T/T SNP5 allele was $6 \mathrm{mg}$ lower when compared to Caucasian men with 'wild-type' $\mathrm{C} / \mathrm{C}$ and $\mathrm{C} / \mathrm{T}$ heterozygous allele, whereas the polymorphism at SNP6 had no effect. The different outcomes of the ARIC study and ours could reflect the difference in race. To that end, the polymorphism at SNP5 which yielded statistically significant results for Caucasian men, had no effect on plasma LDL-c levels in the AA population in the same study. Alternatively, our study could have missed some subtle differences in plasma lipid levels due to its limited size. Further studies in a Chinese population with a larger study group may help to reconcile the different conclusions of these two studies.

The Chinese herbal medicine BBR has been proposed as a new type of cholesterol-lowering drug that works through an action mechanism different from statins, the classic HMG CoA reductase inhibitors. In China, BBR has been used widely as a non-prescription drug to treat diarrhea caused by bacterial infection. The demonstration of a cholesterollowering effect of BBR in a small group of hypercholesterolemic patients suggested the possible use of this compound as a lipid-lowering drug. From this perspective, it is desirable to know the possible influence of SNPs in the 3'UTR on BBR treatment. We used a luciferase reporter to assess the response of three haplotypes to BBR treatment. In transiently transfected HepG2 cells, the normalized luciferase activities were comparable among different haplotypes and the results were consistent in 4 separate transfection assays. This result is different from the results reported by Muallem et al (18). In that study, the reporter Hprt-hrGFPn was used to test the $1-\mathrm{kb}$ proximal region of the LDLR 3'UTR in stably transfected mouse embryonic stem cells. It was shown that the expression of the reporter gene carrying the haplotype GG-C-G-C-A (corresponding to our Type V) sequence was $30 \%$ higher than that containing haplotype A-G-C-A-C-G (our Type I) and $37 \%$ higher than that of haplotype G-G-CG-T-A. It is possible that the use of different reporters and different cell lines contributed to the different results. The discrepancy could also have resulted from a difference in transfection methods: transiently expressed reporters versus stably integrated reporters.

When treated with BBR, the expression of luciferase genes with the 3'UTR containing Type I, IV, and V haplotypes were all elevated to levels of $\sim 1$.7-fold of the control. This suggests that genetic variations at the LDLR 3'UTR do not diminish the effect of this herbal medicine on LDLR expression.

In summary, we have shown that in a Chinese population, common SNPs in the regulatory region of LDLR 3'UTR do not affect LDLR gene expression and the response to BBR treatment.

\section{Acknowledgements}

This study was supported by the Department of Veterans Affairs (Office of Research and Development, Medical Research Service, J.L.), by grant 1RO1 AT002543-01A1 (J.L.) from the National Center for Complementary and Alterative Medicine, by grant U01 HL069757 (R.M.K.), and by a Nanjing Science and Technology Development Research Grant (S.W.). P.S. is supported in part by a European Molecular Biology Organization long-term post-doctoral fellowship.

\section{References}

1. Austin MA, Hutter CM, Zimmern RL and Humphries SE: Genetic causes of monogenic heterozygous familial hypercholesterolemia: A HuGE prevalence review. Am J Epidemiol 160: 407-420, 2004.

2. Defesche JC: Low-density lipoprotein receptor - its structure, function, and mutations. Semin Vasc Med 4: 5-11, 2004.

3. Hobbs HH, Brown MS and Goldenstein JL: Molecular genetics of the LDL receptor gene in familial hypercholesterolemia. Hum Mutat 1: 445-466, 1992.

4. Koivisto U-M, Palvimo JJ, Janne OA and Kontula K: A singlebase substitution in the proximal Sp1 site of the human low density lipoprotein receptor promoter as a cause of heterozygous familial hypercholesterolemia. Proc Natl Acad Sci USA 91: 10526-10530, 1994.

5. Peeters AV, Kotze MJ, Scholtz CL, De Waal LF, Rubinstein DC, Coetzee GA, Zuliani G, Streiff R, Liu J and van der Westhuyzen DR: A 3-basepair deletion in repeat 1 of the LDL receptor promoter reduces transcriptional activity in a South African Pedi. J Lipid Res 39: 1021-1024, 1998.

6. Scholtz CL, Peeters AV, Hoogendijk CF, Thiart R, de Villiers JNP, Hillermann R, Liu J, Marais D and Kotze MJ: Mutation -59c>t in repeat 2 of the LDL receptor promoter: regulation in transcriptional activity and possible allelic interaction in a South African family with familial hypercholesterolaemia. Hum Mol Genet 8: 2025-2030, 1999. 
7. Sun XM, Neuwirth C, Wade DP, Knight BL and Soutar AK: A mutation $(\mathrm{T}-45 \mathrm{C})$ in the promoter region of the low-density lipoprotein (LDL)-receptor gene is associated with a mild clinical phenotype in a patient with heterozygous familial hypercholesterolemia. Hum Mol Genet 4: 2125-2129, 1995.

8. Hoogendijk CF, Scholtz CL, Pimstone SM, Ehrenborg E, Kastelein JJP, Defwsche JC, Thiart R, Plessis L and Kotze MJ: Allelic variation in the promoter region of the LDL receptor gene: analysis of an African-specific variant in the FP2 cisacting regulatory element. Mol Cell Probes 17: 175-181, 2003.

9. Nakahara M, Fujii H, Maloney PR, Shimizu M and Sato R: Bile acids enhance low density lipoprotein receptor gene expression via a MAPK cascade-mediated stabilization of mRNA. J Biol Chem 277: 37229-37234, 2002.

10. Wilson GM, Vasa MZ and Deeley RG: Stabilization and cytoskeletal-association of LDL receptor mRNA are mediated by distinct domains in its 3' untranslated region. J Lipid Res 39: 1025-1032, 1998.

11. Knouff C, Malloy S, Wilder J, Altenburg MK and Maeda N: Doubling expression of the low density lipoprotein receptor by truncation of the 3'-untranslated region sequence ameliorates type III hyperlipoproteinemia in mice expressing the human apoE2 isoform. J Biol Chem 276: 3856-3862, 2001.

12. Kong W, Wei J, Abidi P, Lin M, Inaba S, Li C, Wang Y, Wang Z, Si S, Pan H, Wang S, Wu J, Li Z, Liu J and Jiang JD: Berberine is a promising novel cholesterol-lowering drug working through a unique mechanism distinct from statins. Nat Med 10: 1344-1352, 2004

13. Abidi P, Zhou Y, Jiang J and Liu J: ERK-dependent regulation of hepatic LDL receptor expression by herbal medicine berberine. Arterioscler Thromb Vasc Biol 25: 2170-2176, 2005.
14. Fuster V, Alexander WR and O'Rourke RA (eds): Coronary heart disease. In: Hurst's the Heart. 11th edition, McGraw-Hill Professional, part 6, pp1-10, 2004.

15. Xiao W and Oefner PJ: Denaturing high-performance lipid chromatography: A review. Hum Mutat 17: 439-474, 2001.

16. Strnad P, Lienau TC, Tao G-Z, Lazzeroni LC, Stickel F, Schuppan D and Omary B: Keratin variants associate with progression of fibrosis during chronic hepatitis $\mathrm{C}$ infection. Hepatology 43: 1100-1102, 2006.

17. Dixon DA, Kaplan CD, McIntyre TM, Zimmerman GA and Prescott SM: Post-transcriptional control of cyclooxygenase-2 gene expression. J Biol Chem 275: 11750-11757, 2000.

18. Muallem H, North KE, Kakoki M, Wojczynski MK, Li X, Grove M, Boerwinkle E, Wilhelmsen KC, Heiss G and Maeda N: Quantitative effects of common genetic variations in the 3'UTR of the human LDL-receptor gene and their associations with plasma lipid levels in the Atherosclerosis Risk in Communities study. Hum Genet 121: 421-431, 2007.

19. Chang J, Pan J, Tai D, Huang A, Li P, Ho H, Hsieh H, Chou S, Lin W, Lo E, Chang C, Tseng J, Su M and Lee-Chen G: Identification and characterization of LDL receptor gene mutations in hyperlipidemic Chinese. J Lipid Res 44: 1850-1858, 2003.

20. Knoblauch H, Bauerfeiind A, Krahenbuhl C, Daury A, Rohde K, Bejanin S, Essioux L, Schuster H, Luft FC and Reich JG: Common haplotypes in five genes influence genetic variance of LDL and HDL cholesterol in the general population. Hum Mol Genet 11: 1477-1485, 2002.

21. Hennig BJW, Hellier S, Frodsham AJ, Zhang L, Klenerman P, Knapp S, Wright M, Thomas HC, Thursz M and Hill AVS: Association of low-density lipoprotein receptor polymorphisms and outcome of hepatitis C infection. Genes Immun 3: 359-367, 2002. 